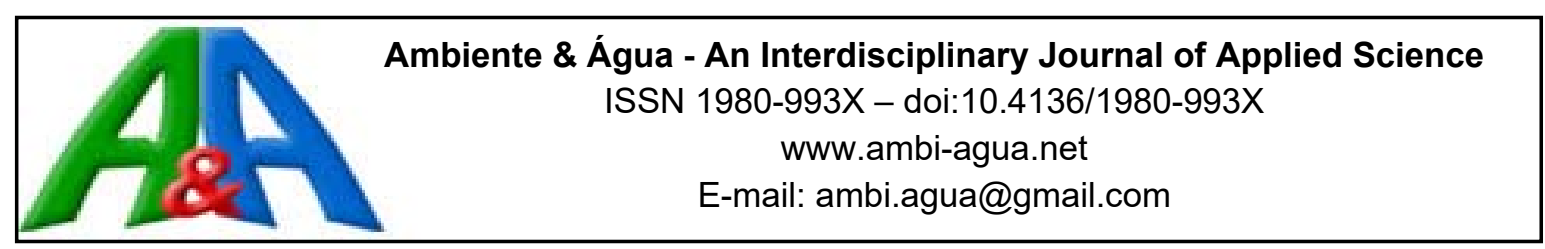

\title{
Variáveis intervenientes na existência de comitês de bacias hidrográficas no Brasil ${ }^{1}$
}

\author{
doi:10.4136/ambi-agua.1828
}

Received: 21 Dec. 2015; Accepted: 26 Jan. 2017

\author{
Alexandre André Feil ${ }^{1 *}$; Vírgilio José Strasburg²; Fernando Rosado Spilki ${ }^{3}$ \\ ${ }^{1}$ Centro Universitário Univates, Lajeado, RS, Brasil \\ Departamento do Centro de Gestão Organizacional \\ ${ }^{2}$ Universidade Federal do Rio Grande do Sul (UFRGS), Porto Alegre, RS, Brasil \\ Departamento de nutrição \\ ${ }^{3}$ Universidade FEEVALE, Novo Hamburgo, RS, Brasil \\ *Autor correspondente: e-mail: alexandre.feil1@gmail.com, \\ vjs.nut@terra.com.br, fernandors@feevale.br
}

\section{RESUMO}

A gestão de bacias hidrográficas por meio de comitês é essencial na mediação entre a oferta e a demanda de água para consumo doméstico, industrial, agrícola, lazer, entre outros usos da água. Uma gestão exitosa pode ocorrer por meio da identificação e compreensão das características socioeconômicas, demográficas, entre outras, e assim formatar as práticas adotadas pelos comitês. Neste contexto, o escopo central deste estudo consistiu em analisar as variáveis intervenientes relacionadas aos aspectos geográficos, demográficos, socioeconômicos e a existência de comitê de bacias. A metodologia consiste na abordagem quantitativa, empregando-se uma pesquisa documental secundária e sua análise ocorreu por meio da aplicação da estatística descritiva e do teste de correlação de Pearson. Os principais resultados evidenciam que a explicação da existência de comitês de bacias hidrográficas relaciona-se com o tamanho da população que promove maior pressão à medida que aumenta. Além disso, evidenciou-se que a existência de comitês de bacias correlaciona-se com: crescimento econômico, quantidade de conflitos pelos múltiplos usos, coleta de esgotos e nível de degradação da qualidade ambiental. Conclui-se, portanto, que de fato existem variáveis intervenientes para a existência de comitês de bacias e que eles são necessários para a gestão eficiente destas variáveis.

Palavras-chave: demográficos, geográficos, Pearson test, recursos hídricos, saneamento, socioeconômicos.

\section{Intervening variables in the existence of watershed committees in Brazil}

\begin{abstract}
Watershed management through committees is essential in mediating between water supply and demand for domestic consumption, as well as industrial, agricultural, leisure, and other uses of water. Successful management can occur through the identification and

\footnotetext{
${ }^{1}$ Uma versão deste artigo foi publicada como resumo expandido no Seminário de Pós-Graduação, 2014 com o título: "Fatores que influenciam na consolidação de Comitês das Bacias Hidrográficas brasileiras". Novo Hamburgo RS: Feevale, 2014. v. 6. p. 175-80. ISSN 2358-4599
} 
comprehension of socioeconomic and demographic characteristics, among others, and therefore, these help shape the practices adopted by the committees. In this context, the central objective of this study was to analyse the intervening variables related to the geographic, demographic, socioeconomic aspects and the existence of a basin committee. The methodology consisted of a quantitative approach, using previously published documents and analysis applying descriptive statistics and the Pearson correlation test. The main results showed that the existence of a watershed committee is correlated with the size of the population, which promotes greater pressure as it increases. In addition, it was observed that the creation of committees is related to economic growth, number of conflicts for multiple uses, sewage collection and level of environmental quality degradation. We conclude, therefore, that in fact there are intervening variables for the existence of river basin committees and that the committees are necessary for the efficient management of those variables.

Keywords: geographic, demographic, Pearson test socioeconomic, sanitation, water resources.

\section{INTRODUÇÃO}

A gestão de Bacias Hidrográficas (BHs) por meio de Comitês de Bacias Hidrográficas (CBHs) é essencial na solução de conflitos, principalmente pela degradação e distribuição equitativa da água aos inúmeros interessados. A gestão dos recursos hídricos deve estar direcionada aos recursos naturais em função do crescimento de cada componente na $\mathrm{BH}$, pois, as participações públicas envolvidas nos $\mathrm{CBHs}$ possuem muitas perspectivas, interesses e alternativas de uso dos recursos hídricos em função da diversidade, renda, prioridades, localização, posições, entre outras (Sanchez-Roman et al., 2009).

As BHs compreendem uma área, na qual após a precipitação, a água escorre através de redes de córregos pelo processo natural, até desaguar em um rio, lago, represa ou oceano, que pode distanciar-se a alguns ou a milhares de hectares da área da superfície (Datta, 2015). O desenvolvimento de BHs compreende um instrumento para obtenção de melhorias na distribuição, resolução de conflitos de interesses, produtividade, preservação e conservação da água, onde a degradação de recursos, principalmente os hídricos compreendem um grave problema (Hope, 2007). Neste sentido, a gestão de BHs é um instrumento - em função da disponibilidade da base de dados e informações, clara definição dos diretos de uso, controles de impactos aos recursos hídricos e o processo de tomada de decisão - essencial na preservação da qualidade e quantidade, utilização racional e integrada dos recursos hídricos e pela influência de diversas variáveis intervenientes (Silva et al., 2008).

Os CBHs possuem como escopo a gestão destes recursos hídricos de uma unidade territorial denominada de $\mathrm{BH}$ por meio da participação pública. Esta participação pública em função da diversidade de membros agrega diversos benefícios, pois possuem diferentes relações com o meio ambiental, em especial, com a água (Hajkowicz, 2007; Perkins, 2011; Malheiros et al., 2013).

A participação pública abrange indivíduos ou grupos afetados positiva e negativamente pela disponibilidade de recursos hídricos cujos interesses influem ou sejam influenciados pelo projeto proposto, programa, plano ou política, as quais vinculam-se ao processo de tomada de decisão (André et al., 2006). Liu et al. (2008), Kaplowitz e Witter (2008) e Perkins (2011) salientam que a incorporação das comunidades locais no processo de planejamento melhora o sucesso da gestão dos recursos hídricos pelos CBHs. Os objetivos da participação pública na gestão dos CBHs, segundo André et al. (2006), compreendem: a) promover a justiça, equidade e colaboração; b) informar as medidas e suas consequências aos diversos stakeholders; c) promover a aprendizagem recíproca do público participante; e d) reduzir os impactos negativos e mitigar conflitos (Porto et al., 1999). Portanto, a forma organizada dessa participação ocorre mediante a estrutura dos CBHs. 
Os processos de participação na gestão de BHs por meio de CBHs possuem aspectos positivos e negativos (Korff et al., 2010). Estes autores destacam que os aspectos positivos se vinculam à legitimidade das tomadas de decisões pelas partes interessadas e mais adequadas em função da consideração de diferentes conhecimentos, maior chance da implantação das decisões e menos oposições, e o aumento da aprendizagem entre os participantes. Os aspectos negativos vinculam-se a decepção dos interessados quando as expectativas não são cumpridas ou incertas, a tomada de decisão em aspectos não importantes e não representados durante o processo e a oposição de implementação de decisões.

No Brasil, a lei 9.433/97 institui a Política Nacional dos Recursos Hídricos (PNRH) criou o Sistema Nacional de Gerenciamento de Recursos Hídricos (SNGRH), e este gerenciamento dos ocorre por meio de decisões locais os quais são denominados de CBHs. Porto e Porto (2008) salientam que esta legislação propicia um destaque ao Brasil, colocando-o entre os países mais avançados no setor de recursos hídricos. O SNGRH, criado pela lei 9433/97, possui os objetivos frente aos recursos hídricos de: a) coordenar a gestão integrada; b) arbitrar os conflitos; c) implementar a PNRH; d) planejar, regular e controlar o uso, a preservação e sua recuperação; e e) promover a cobrança pelo seu uso.

A descentralização desta gestão em nível local ainda carece de uma evolução institucional no país (Porto e Porto, 2008). Estes autores ainda destacam que a lei 9433/97 contribui para um novo paradigma referente a gestão dos recursos hídricos, sendo que a falta desta gestão pode refletir efeitos perversos para toda sociedade brasileira. Cabe destacar que o Brasil ainda estabelece a existência de CBHs transjurisdicionais com integrantes governamentais e da sociedade civil com a finalidade de discutir e decidir questões relativas à gestão da água em cada BH (Perkins, 2011). Estas questões, segundo Datta (2015) abrangem os elementos de solo e conservação da água, incluindo a melhoria de infraestrutura agrícola e social, mercado e acesso ao crédito, e introduz novas tecnologias agrícolas para o desenvolvimento das BHs.

Nesta lógica, este estudo possui como objetivo central analisar se as variáveis intervenientes relacionados aos dados geográficos, demográficos, socioeconômicos e de saneamento possuem correlação com a existência de CBHs. Este propósito justifica-se pela relevância das particularidades quanto aos aspectos geográficos das BHs e frente à distribuição de CBHs no território nacional. Além disto, salienta-se que há carência de um mapeamento comparativo que associe as questões da existência e distribuição dos CBHs entre as Unidades Federativas (UF) brasileiras em relação aos aspectos geográficos, demográficos, socioeconômicos e saneamento.

Kaplowitz e Witter (2008) e Mahmoud et al. (2011) reforçam esta justificativa, destacando que com o prolongamento da escassez hídrica exercendo pressões sobre os sistemas de gestão e distribuição de água existentes torna-se essencial a identificação e análise dos fatores que podem alterar o gerenciamento do consumo para uma eficiente gestão, incluindo as BHs. O sucesso na gestão de BHs, segundo Brody et al. (2005), é realizado por meio de diferentes grupos de stakeholders, mas através de uma melhor compreensão das características socioeconômicas, demográficas e demais variáveis intervenientes.

\section{MATERIAL E MÉTODOS}

Este estudo, quanto à abordagem do problema caracteriza-se como quantitativo, quanto ao procedimento técnico vincula-se a pesquisa documental referente a coleta dos dados e causal em relação a análise das informações coletas. Neste sentido, a pesquisa causal compreende a análise das variáveis intervenientes, por meio da estatística, na explicação de um fenômeno, que neste estudo direciona-se a explicação da existência de $\mathrm{CBH}$ para a gestão dos recursos hídricos. 


\subsection{Coleta e tabulação dos dados}

A coleta dos dados foi realizada pela pesquisa documental secundária abrangendo aspectos geográficos, demográficos, socioeconômicos e de saneamento (Quadro 1).

Quadro 1. Dados coletados e seleção das variáveis.

\begin{tabular}{|lllll|}
\hline Tipo de dados & \multicolumn{1}{c}{ Variáveis } & \multicolumn{1}{c}{ Medida } & \multicolumn{1}{c|}{ Fonte } & Ano base \\
\hline \multirow{5}{*}{ Geográficos } & $-\mathrm{n}^{\circ}$ de municípios por UF & Número & $(\mathrm{CBH}, 2014)$ & 2011 \\
& - Área da BH por UF & $\mathrm{km}^{2}$ & $(\mathrm{CBH}, 2014)$ & 2011 \\
& - CBH por UF & Número & $(\mathrm{CBH}, 2014)$ & 2011 \\
& - Municípios c/CBHs & Número & $(\mathrm{CBH}, 2014)$ & 2011 \\
& - BH por UF & Número & $(\mathrm{CBH}, 2014)$ & 2011 \\
& - Municípios abrangidos por BH & Número & (CBH, 2014) & 2011 \\
\hline \multirow{2}{*}{ Demográficos } & - habitantes por UF & Número & (IBGE, 2013) & 2013 \\
& - habitantes por BH & Número & (CBH, 2014) & 2011 \\
\hline \multirow{5}{*}{ Sócioeconômicos } & - PIB & Percentual & (SIDRA, 2014) & 2011 \\
& - IDHM & Coeficiente & (PNUD et al., 2010) & 2010 \\
& - Renda per capita & Hectares & (SIDRA, 2014) & 2014 \\
& - Índice de degradação ambiental & Reais & (DATASUS, 2014) & 2010 \\
& - Conflitos pela água & Número & (Pinto et al., 2014) & 2014 \\
\hline \multirow{5}{*}{ Saneamento } & - Perda de água na distribuição & Percentual & (TRATABRASIL, 2014) & 2014 \\
& - Tratamento de esgoto & Percentual & (TRATABRASIL, 2014) & 2014 \\
& - Coleta de esgoto & Percentual & (TRATABRASIL, 2014) & 2014 \\
& - Rede de água & Percentual & (TRATABRASIL, 2014) & 2014 \\
\hline
\end{tabular}

Nota: *2014, 2013 e 2012; PIB: Produto Interno Bruto; IDHM: Índice de Desenvolvimento Humano.

Fonte: CBH (2014); IBGE (2013); SIDRA (2014); PNUD et al. (2010); DATASUS (2014); Pinto et al. (2014); CPT (2014); TRATABRASIL (2014).

Esta pesquisa documental foi realizada de março a abril de 2014, em sítios oficiais de internet descritos na coluna fonte do Quadro 1. A seleção das variáveis (Quadro 1) ocorreu em função da disponibilidade de dados e pela aderência aos possíveis fatores que influenciam na existência de CBHs nas diferentes UFs brasileiras. Salienta-se como limitação desta pesquisa documental secundária que podem existir outras variáveis que influenciam no estabelecimento de CBHs nas regiões brasileiras, mas que não estão documentadas ou compreendem dados confiáveis. Após a coleta dos dados, estes foram tabulados por meio de planilhas eletrônicas (Software Microsoft Office Excel), onde na primeira linha inseriu-se a descrição das variáveis por tipo de dados e na primeira coluna da descrição de cada UF. As UFs que integraram este estudo limitam-se as que possuem CBHs.

\subsection{Método de análise}

O método estatístico empregado para a apuração das correlações foi o coeficiente de correlação de Pearson, pois os dados utilizados são paramétricos, e suas medidas escalares são contínuas (Witte e Witte, 2005). O coeficiente de correlação de Pearson está compreendido entre -1 e 1, e a intensidade de correlação é avaliada em: Nula, quando a correlação for 0; Fraca, quando estiver entre 0 e 0,3 ; regular, quando entre 0,31 e 0,6 ; Forte, quando entre 0,61 e 0,9 ; Muito Forte, quando entre 0,91 e 99; e plena, quando apresentar uma correlação igual a 1 (Callegari-Jaques, 2003). O software empregado para correlacionar as variáveis foi o Statistical Package for Social Science for Windows (IBM SPSS). Após apresentar o material e método na sequência apresenta-se os resultados e discussão. 


\section{RESULTADOS E DISCUSSÃO}

Os resultados oriundos da coleta dos dados descrita no Quadro 1, tais como as variáveis geográficas, demográficas, socioeconômicas e de saneamento das UFs brasileiras que possuem CBHs, estão evidenciados na Tabela 1. As UFs estão distribuídas de acordo com as regiões brasileiras: Centro-Oeste (4 UFs), Nordeste (8 UFs), Norte (1 UF), Sudeste (4 UFs) e Sul (3UFs)

Tabela 1. Informações geográficas, demográficas, socioeconômicas e de saneamento.

\begin{tabular}{|c|c|c|c|c|c|c|c|c|c|c|c|c|c|c|c|c|c|c|c|}
\hline \multirow[b]{2}{*}{ UF } & \multicolumn{6}{|c|}{ Geográficos } & \multicolumn{2}{|c|}{ Demográficos } & \multicolumn{7}{|c|}{ Sócio econômicos } & \multicolumn{4}{|c|}{ Saneamento } \\
\hline & 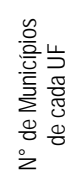 & 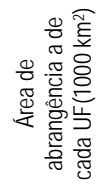 & 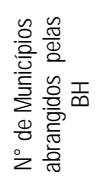 & 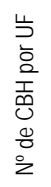 & 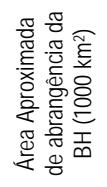 & 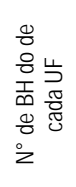 & 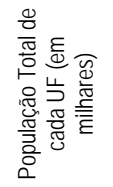 & 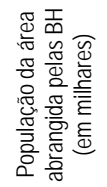 & 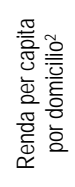 & $\begin{array}{l}\sum_{\underline{\underline{I}}}^{m} \\
\underline{\underline{\underline{n}}}\end{array}$ & $\frac{\underline{m}}{\alpha}$ & 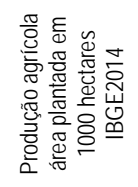 & 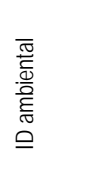 & 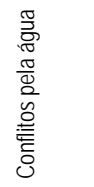 & 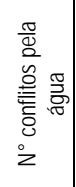 & 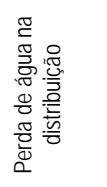 & 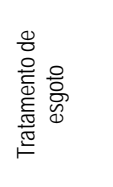 & 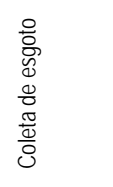 & 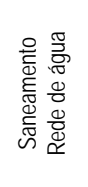 \\
\hline Distrito Federal & 1 & 5,8 & 1 & 3 & 5,8 & 7 & 2789,8 & 2.616 & 1.665 & 0,824 & 164482 & 172,9 & 44,65 & 0,0 & 0 & 37,23 & 66,13 & 82,73 & 98,20 \\
\hline Goiás & 246 & 340,1 & 37 & 3 & 12,4 & 11 & 6434,0 & 2.009 & 785 & 0,735 & 111269 & 6087,3 & 80,14 & 1,0 & 2 & 28,78 & 44,93 & 41,51 & 85,62 \\
\hline Mato Grosso & 141 & 903,4 & 54 & 7 & 109,3 & 27 & 3182,1 & 1.204 & 735 & 0,725 & 71418 & 13542,0 & 68,20 & 4,3 & 6 & 47,17 & 23,85 & 17,72 & 86,23 \\
\hline Mato Grosso do Sul & 79 & 357,1 & 20 & 2 & 41,6 & 15 & 2587,3 & 204 & 785 & 0,729 & 49242 & 4551,7 & 80,19 & 0,0 & 0 & 32,92 & 32,76 & 36,47 & 85,75 \\
\hline Alagoas & 102 & 27,8 & 66 & 5 & 14,4 & 14 & 3300,9 & 3.563 & 421 & 0,631 & 28540 & 571,8 & 39,76 & 1,3 & 2 & 46,12 & 18,52 & 18,83 & 76,46 \\
\hline Bahia & 417 & 564,7 & 366 & 10 & 372,5 & 25 & 15044,1 & 9.785 & 481 & 0,66 & 159869 & 3584,3 & 69,62 & 18,3 & 26 & 41,58 & 46,56 & 31,02 & 77,43 \\
\hline Ceará & 184 & 148,9 & 193 & 10 & 131,5 & 12 & 8778,6 & 7.762 & 446 & 0,682 & 87982 & 1012,1 & 43,57 & 3,0 & 1 & 36,52 & 32,22 & 25,32 & 69,75 \\
\hline Paraíba & 223 & 56,5 & 145 & 3 & 25,7 & 19 & 3914,4 & 2.505 & 462 & 0,658 & 35444 & 332,7 & 54,64 & 1,7 & 0 & 36,18 & 34,02 & 24,54 & 75,6 \\
\hline Pernambuco & 185 & 98,1 & 170 & 7 & 39,6 & 33 & 9208,6 & 5.503 & 509 & 0,673 & 104394 & 803,7 & 32,46 & 6,0 & 8 & 53,69 & 26,38 & 19,68 & 70,89 \\
\hline Piauí & 224 & 251,6 & 94 & 1 & 75,0 & 12 & 3184,2 & 667 & 408 & 0,646 & 24607 & 1443,7 & 72,35 & 0,3 & 0 & 51,82 & 8,30 & 6,64 & 67,12 \\
\hline Rio Grande do Norte & 167 & 52,8 & 85 & 3 & 18,2 & 16 & 3374,0 & 1.831 & 532 & 0,684 & 36103 & 198,7 & 59,81 & 0,7 & 1 & 55,26 & 21,09 & 21,54 & 81,37 \\
\hline Sergipe & 75 & 21,9 & 61 & 3 & 9,5 & 8 & 2195,7 & 1.644 & 508 & 0,665 & 26199 & 284,8 & 50,13 & 0,3 & 0 & 59,27 & 22,39 & 15,25 & 83,05 \\
\hline Tocantins & 139 & 277,7 & 50 & 7 & 56,2 & 30 & 1478,2 & 557 & 572 & 0,699 & 18059 & 1033,2 & 78,40 & 2,0 & 2 & 34,34 & 17,65 & 14,71 & 75,45 \\
\hline Espírito Santo & 78 & 46,1 & 75 & 13 & 26,2 & 14 & 3839,4 & 2.312 & 795 & 0,74 & 97693 & 131,0 & 53,23 & 3,0 & 5 & 34,39 & 32,36 & 41,82 & 80,9 \\
\hline Minas Gerais & 853 & 586,5 & 1083 & 38 & 557,5 & 43 & 20593,4 & 18.779 & 733 & 0,731 & 386156 & 4329,4 & 55,73 & 14,0 & 26 & 33,46 & 32,76 & 74,22 & 86,97 \\
\hline Rio de Janeiro & 92 & 43,8 & 110 & 10 & 40,5 & 10 & 16369,2 & 15.660 & 993 & 0,761 & 462376 & 122,0 & 55,19 & 5,7 & 7 & 30,82 & 34,32 & 62,59 & 89,15 \\
\hline São Paulo & 645 & 248,2 & 829 & 22 & 248,4 & 22 & 43663,7 & 39.809 & 1.037 & 0,783 & 1349465 & 7503,7 & 43,92 & 1,0 & 0 & 34,34 & 53,34 & 87,36 & 95,85 \\
\hline Paraná & 399 & 199,3 & 476 & 11 & 183,5 & 16 & 10997,5 & 10.244 & 871 & 0,749 & 239366 & 10587,9 & 55,48 & 2,0 & 3 & 33,35 & 63,75 & 60,00 & 91,15 \\
\hline Rio Grande do Sul & 295 & 95,7 & 672 & 25 & 271,2 & 25 & 6634,3 & 10.603 & 940 & 0,746 & 263633 & 8871,2 & 42,31 & 2,0 & 2 & 37,23 & 12,58 & 29,15 & 84,06 \\
\hline Santa Catarina & 497 & 281,7 & 321 & 17 & 78,0 & 24 & 11164,0 & 5.129 & 967 & 0,774 & 169050 & 1545,7 & 19,79 & 2,3 & 3 & 33,71 & 19,58 & 16,03 & 86,02 \\
\hline
\end{tabular}

Nota: ${ }^{1}$ Produto Interno Bruto de cada Estado, em 2011, valores em (R\$1.000,00).

2 Renda per capita por domicílio corresponde à média mensal, em 2010, e em R\$1.000,00.

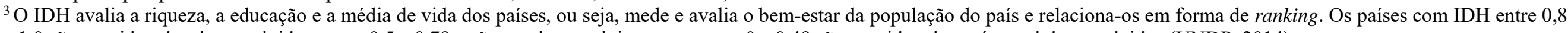
e 1,0 são considerados desenvolvidos, entre 0,5 e 0,79 estão em desenvolvimento, e entre 0 e 0,49 são considerados países subdesenvolvidos (UNDP, 2014).

Rev. Ambient. Água vol. 12 n. 2 Taubaté - Mar. / Apr. 2017 
A correlação de Pearson realizou-se com base na Tabela 1, e seus resultados estão evidenciados na Tabela 2. As correlações revelam que existe uma forte correlação, conforme classificação de Callegari-Jaques (2003), entre o número de CBH com: o número de municípios de cada UF $\left(p=0,806^{* *}\right)$; a população total de cada UF (habitantes) $\left(p=0,613^{* *}\right)$; a área aproximada de abrangência da $\mathrm{BH}\left(p=0,826^{* *}\right)$; o número de municípios abrangidos pelas $\mathrm{BH}\left(p=0,919^{* *}\right)$; e a população da área abrangida pelas $\mathrm{BH}\left(p=0,664^{* *}\right)$. Estes resultados apontam que o número de $\mathrm{CBHs}$ existentes podem ser relacionados as regiões com maior concentração populacional.

Tabela 2. Correlação de Pearson entre variáveis socioeconômicas e geográficas de BHs brasileiras.

\begin{tabular}{|c|c|c|c|c|c|c|c|c|c|c|c|c|c|c|c|c|c|c|c|}
\hline & 1 & 2 & 3 & 4 & 5 & 6 & 7 & 8 & 9 & 10 & 11 & 12 & 13 & 14 & 15 & 16 & 17 & 18 & 19 \\
\hline 1 & 1 & & & & & & & & & & & & & & & & & & \\
\hline 2 & 0,416 & 1 & & & & & & & & & & & & & & & & & \\
\hline 3 & $0,730 * *$ & 0,163 & 1 & & & & & & & & & & & & & & & & \\
\hline 4 & $0,575 * *$ & 0,047 & $0,949 * *$ & 1 & & & & & & & & & & & & & & & \\
\hline 5 & 0,034 & $-0,092$ & 0,241 & 0,405 & 1 & & & & & & & & & & & & & & \\
\hline 6 & 0,19 & 0,036 & 0,363 & $0,498^{*}$ & $0,942 * *$ & 1 & & & & & & & & & & & & & \\
\hline 7 & $0,806^{* *}$ & 0,259 & $0,613 * *$ & $0,543^{*}$ & 0,222 & 0,371 & 1 & & & & & & & & & & & & \\
\hline 8 & $0,834 * *$ & $0,515^{*}$ & $0,567 * *$ & 0,416 & 0,008 & 0,101 & $0,826^{* *}$ & 1 & & & & & & & & & & & \\
\hline 9 & $0,686^{* *}$ & 0,077 & $0,975 * *$ & $0,964 * *$ & 0,28 & 0,382 & $0,664 * *$ & $0,585^{* *}$ & 1 & & & & & & & & & & \\
\hline 10 & $0,909 * *$ & 0,258 & $0,733 * *$ & $0,650 * *$ & 0,149 & 0,269 & $0,919 * *$ & $0,887 * *$ & $0,775 * *$ & 1 & & & & & & & & & \\
\hline 11 & $0,638 * *$ & $0,541^{*}$ & 0,303 & 0,163 & $-0,168$ & $-0,036$ & $0,649 * *$ & $0,661 * *$ & 0,278 & $0,611 * *$ & 1 & & & & & & & & \\
\hline 12 & $-0,444 *$ & $-0,056$ & $-0,4$ & $-0,451^{*}$ &,$- 602 * *$ & $\begin{array}{c}- \\
n 77 n * *\end{array}$ & $-0,618^{* *}$ & $-0,34$ & $-0,44$ & $-0,543 *$ & $-0,093$ & 1 & & & & & & & \\
\hline 13 & 0,343 & $0,637 * *$ & 0,257 & 0,299 & 0,19 & 0,307 & 0,307 & 0,403 & 0,283 & 0,397 & 0,286 & $-0,474 *$ & 1 & & & & & & \\
\hline 14 & $-0,294$ & $-0,111$ & $-0,314$ & $-0,33$ & $-0,474 *$ & $\stackrel{-}{n \text { โอ0*** }}$ & $-0,377$ & $-0,233$ & $-0,32$ & $-0,284$ & $-0,056$ & $0,517^{*}$ & $-0,2$ & 1 & & & & & \\
\hline 15 & 0,169 & 0,003 & 0,396 & 0,403 & $0,574 * *$ & $0,537^{*}$ & 0,032 & 0,153 & 0,373 & 0,171 & $-0,204$ & $-0,27$ & 0,216 & $-0,450^{*}$ & 1 & & & & \\
\hline 16 & 0,389 & $-0,022$ & $0,641 * *$ & $0,702 * *$ & $0,722 * *$ & $0,723 * *$ & $0,456^{*}$ & 0,381 & $0,681 * *$ & $0,503^{*}$ & $-0,004$ & $-0,507^{*}$ & 0,195 & $-0,543^{*}$ & $0,781^{* *}$ & 1 & & & \\
\hline 17 & 0,223 & 0,083 & 0,408 & $0,544^{*}$ & $0,848^{* *}$ & $0,851 * *$ & 0,303 & 0,152 & 0,441 & 0,304 & $-0,099$ & $-0,641 * *$ & 0,399 & $-0,42$ & $0,630 * *$ & $0,777 * *$ & 1 & & \\
\hline 18 & $-0,178$ & 0,429 & $-0,246$ & $-0,229$ & $-0,224$ & $-0,190$ & $-0,338$ & $-0,005$ & $-0,274$ & $-0,266$ & $-0,080$ & 0,361 & 0,215 & $-0,108$ & 0,027 & $-0,081$ & $-0,095$ & 1 & \\
\hline 19 & $0,502 *$ & $0,509^{*}$ & 0,315 & 0,078 & $-0,184$ & $-0,148$ & $0,465^{*}$ & $0,738 * *$ & 0,259 & $0,449 *$ & $0,582 * *$ & $-0,006$ & 0,067 & $-0,076$ & 0,126 & 0,143 & $-0,098$ & 0,071 & 1 \\
\hline
\end{tabular}

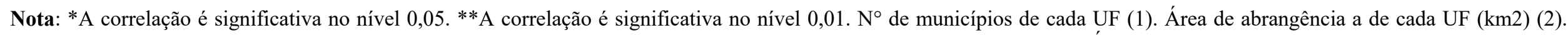

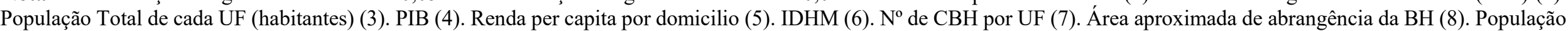

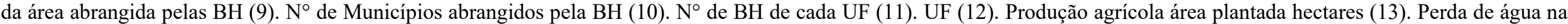
distribuição (14). Tratamento de esgoto (15). Coleta de esgoto (16). Rede de água (17). ID ambiental (18). Conflitos pela água (19). 
O aumento da concentração populacional em determinada área, pode gerar um conflito pela distribuição equitativa de água da $\mathrm{BH}$, pois esta abastece as residências e indústrias urbanas, bem como a produção agrícola rural, quando for o caso. Barreto et al. (2010) destacam que a água é utilizada como estratégia na administração de conflitos e proteção ambiental, o que justificaria o estabelecimento da gestão de $\mathrm{BHs}$ por meio de $\mathrm{CBH}$, pois busca o equilíbrio entre a demanda e o consumo humano, dessedentação animal e irrigação. Neste sentido, Mahmoud et al. (2011) em seu estudo revela que o fator crescimento populacional obriga os gestores de $\mathrm{BHs}$ e os stakeholders a tomarem decisões críticas sob diferentes graus de incertezas. Gaddis et al. (2010) salientam que a gestão de BHs por CBHs afeta e é afetada pela sociedade, principalmente em relação à poluição.

A institucionalização de CBHs possui correlação moderada com regiões em que há a coleta de esgoto $\left(p=0,456^{*}\right)$, porém com os demais aspectos de saneamento relacionados a perda de água na distribuição, tratamento de esgoto e a disponibilidade da rede de água não apresentou correlação significativa. Entretanto, esse achado não corrobora com estudos de Liu et al. (2008) que revelam que a gestão hídrica, principalmente em regiões de pobreza onde não há investimentos em recursos naturais, é necessária para resolver adequadamente os problemas com a distribuição de água. Os resultados de Abers e Jorge (2005) afirmam que a criação de comitês, na maioria dos casos, relaciona-se na solução de problemas na $\mathrm{BH}$, tais como, os ambientais, conflitos entre usos da água e a incidência de eventos críticos.

Esta reflexão corrobora com o que é postulado por Said et al. (2006) afirmando que a deterioração da qualidade e escassez de água são os propulsores do desenvolvimento de estratégias de gestão das BHs por meio de CBHs. E ainda salientam que um dos propósitos da gestão de BHs vincula-se à compreensão das ações necessárias no restabelecimento da qualidade da água. Campos (2004) exemplifica que a criação das Bacias Hidrográficas dos Rios Piracicaba, Capivari e Jundiaí/SP ocorreu em função da degradação e da escassez de águas comprometerem o desenvolvimento socioeconômico desta região, portanto o $\mathrm{CBH}$ teve como objetivo a preservação e restauração ecológica. Os gestores, diante dos diversos interesses e necessidades dos usuários, podem ter dificuldades para compreender os diferentes objetivos destes grupos (Kaplowitz e Witter, 2008). Portanto, a gestão de BHs destina-se a proteger os recursos hídricos superficiais e subterrâneos, e para isto, centra-se na gestão integrada e participativa dos recursos da água e do solo (Ferreyra et al., 2008).

A quantidade de $\mathrm{CBHs}$ também apresentou uma correlação significativa moderada $\left(p=0,543^{*}\right)$ com o PIB da cada UF, conforme classificação de Callegari-Jaques (2003), mostrando que quanto maior o PIB do estado, maior é o número de CBHs estabelecidos e funcionando. Neste sentido, esta informação revela que o processo de instalação de CBHs tem uma relação com o PIB, pois na medida em que ocorre um crescimento econômico, poderá refletir-se na disponibilidade da água.

Sanchez-Roman et al. (2009) salientam que o desenvolvimento econômico demanda enormes quantidades de água. Said et al. (2006) corrobora com esta afirmação, destacando que o aumento da gestão hídrica e a participação pública, nestes comitês, está vinculada ao estímulo econômico. Para Falkenmark e Molden (2008) o crescimento econômico da população implica no crescimento da demanda pela água e de alimentos. A região Sudeste do Brasil enfrenta os principais problemas de recursos hídricos voltados à questão da escassez pela poluição e o uso excessivo das águas nas áreas urbanas e industrializadas, seus conflitos de uso e as constantes inundações (Porto et al., 1999). Barreto et al. (2010) acrescenta que a associação entre a falta hídrica e a exploração econômica desordenada provocam a degradação e desertificação do solo, e a redução de espécies de vegetais e animais.

O número de CBHs com a variável UF também apresentou uma correlação significativa forte $\left(p=-0,618^{* *}\right)$. Este resultado revela que as regiões brasileiras do sul e sudeste possuem 
maior quantidade de CBHs e, portanto, maior participação pública para a realização da gestão das BHs em relação às regiões do Nordeste, Centro Oeste e Norte. Destaca-se que para a região Norte as informações acerca de CBHs foi de difícil obtenção quando comparada a outras UFs, apesar desta possuir a maior BH brasileira, a do Amazonas com 7,05 milhões de km² (Da Costa et al., 2013).

Este resultado, das regiões do sul e sudeste do Brasil apresentarem maior quantidade de CBHs, também é explicado por Abers e Jorge (2005) que apontam que os governos estaduais foram os responsáveis pela criação de $\mathrm{CBHs}$ em todas as BHs dos estados de São Paulo e do Rio Grande do Sul. Entretanto, os mesmos autores ainda destacam que a criação de CBHs nos estados de Minas Gerais e no Ceará, ocorreu de forma mais seletiva, ou seja, sua criação ocorreu nas BHs de maior interesse para os órgãos governamentais. Watanabe et al. (2014) afirmam que a formação de CBHs no Rio Grande do Sul foi influenciada por decisões locais e consideradas como particulares em cada região do estado.

A quantidade de CBHs também apresentou uma correlação moderada $\left(p=0,465^{*}\right)$ com a quantidade de conflitos pela água em cada UF. Braga et al. (2008) também apontam que nas regiões do Brasil, onde há intensa ocupação do território, tem gerado conflitos pelo uso da água. Portanto, a intensa ocupação populacional em determinada região promove conflitos em relação ao uso das águas pela sua quantidade e qualidade, o que exige a necessidade de institucionalização de um CBH para gerenciar os conflitos entre os diversos usuários.

\section{CONCLUSÃO}

O escopo principal deste estudo foi verificar se as variáveis intervenientes relacionadas aos dados geográficos, demográficos, socioeconômicos e de saneamento possuem correlação com a criação de $\mathrm{CBHs}$. O estabelecimento de $\mathrm{CBH}$ vincula-se com as $\mathrm{BHs}$ com os conflitos no uso da água pelos diversos stakeholders, assim como, também com o tamanho populacional e crescimento econômico (PIB).

A existência de CBHs no Brasil, advém do vasto aglomerado de habitantes que necessitam de grande quantidade de água, para consumo humano e animal, produção de alimentos, de produtos na indústria, recreação, entre outros. Esta por sua vez, cria uma necessidade de demanda muito elevada e pode provocar até a escassez de água, e também, ao mesmo tempo, a degradação através dos resíduos orgânicos sólidos e líquidos, químicos, erosão do solo, entre outros. A partir desta escassez e pela falta de qualidade de água, pode ocorrer uma estagnação e ou decrescimento da situação econômica.

Portanto, é essencial na gestão de BHs por meio de CBHs a participação pública local e específica, pois estes se relacionam tanto na criação e no sucesso da implantação de estratégias principalmente vinculados a degradação ambiental e conflitos de múltiplos usos. Considera-se vital a gestão dos recursos hídricos, especialmente num país com tantas particularidades e dimensões continentais como o Brasil. E, ainda, recomenda-se que nas BHs estruturem-se CBHs antes de sua degradação, pois neste caso, a gestão torna-se mais eficiente e os conflitos mais amenos, contribuindo assim, no estabelecimento da resiliência do sistema ambiental.

\section{AGRADECIMENTOS}

Ao Centro Universitário Univates, Universidade Federal do Rio Grande do Sul e ao Programa de Pós-Graduação em Qualidade Ambiental da Universidade Feevale pelo apoio à pesquisa.

\section{IPABH}




\section{REFERÊNCIAS}

ABERS, R. N.; JORGE K. D. Descentralização da Gestão da Água: Por que os comitês de bacia estão sendo criados? Ambiente \& Sociedade, v. 8, p. 1-26, 2005.

ANDRÉ, P.; ENSERIK, B.; CONNOR, D.; CROAL, P. Public participation: international best practice principles Fargo: . International Association for Impact, 2006.

BARRETO, J. F.; DANTAS NETO, J.; FARIAS, S. A. R. Avaliação socioeconômica e hídrica dos municípios da sub-bacia hidrográfica do rio Taperoá, PB. Qualit@s Revista Eletrônica, v. 9, n. 1, p. 1-13, 2010. http://dx.doi.org/10.18391/qualitas.v9i1.585

BRAGA, P. F. B.; FLECHA, R.; PENA, S. D.; KELMAN, J. Pacto federativo e gestão de águas. Estudos Avançados, v. 22, n. 63, p. 17-42, 2008. http://dx.doi.org/10.1590/S0103-40142008000200003

BRODY, S. D.; HIGHFIELD, W.; PECK, M. B. Exploring the mosaic of perceptions for water quality across watersheds in San Antonio, Texas. Landscape and Urban $\begin{array}{llllll}\text { Planning, } & \text { v. } 73, \quad \text { n. } 2 / 3, \quad \text { p. 200-214, }\end{array}$ http://dx.doi.org/10.1016/j.landurbplan.2004.11.010CALLEGARI-JACQUES， S. M. Bioestatística: princípios e aplicações. Porto Alegre: Artmed, 2003. 256 p.

CAMPOS, V. N. D. O. Novos arranjos, velhos problemas. Revista Electrónica de la REDLACH, v. 1, n. 1, p. 63-68, 2004.

COMITÊS DE BACIAS HIDROGRÁFICAS. Website. 2014. Disponível em: http://www.cbh.gov.br/. Acesso em: 17 set. 2014.

COMISSÃO PASTORAL DA TERRA - CPT. Conflitos pela água. 2014. Disponível em: https://goo.gl/SKnID1. Acesso em: 11 dez. 2014.

DA COSTA, L. J.; MOURA, R. G.; OLIVEIRA, J. M. Análise multitemporal das mudanças do uso do solo ocorridas entre os anos 1986 e 2010 na bacia do rio dos Sinos-Rio Grande Sul-Brasil. In: SIMPÓSIO BRASILEIRO DE SENSORIAMENTO REMOTO - SBSR, 16., 2013, Foz do Iguaçu. Anais... São José dos Campos: INPE, 2013. p. 7438-7444.

DATTA, N. Evaluating Impacts of Watershed Development Program on Agricultural Productivity, Income, and Livelihood in Bhalki Watershed of Bardhaman District, West Bengal. World Development, v. 66, p. 443-456, 2015. http://dx.doi.org/10.1016/j.worlddev.2014.08.024

DATASUS. Indicadores socioeconômicos. 2014. Disponível em: http://tabnet.datasus.gov.br/cgi/tabcgi.exe?idb2011/b08a.def. Acesso em: 12 set. 2014.

FALKENMARK, M.; MOLDEN, D. Wake up to realities of river basin closure. International Journal of Water Resources Development, v. 24, p. 201-215, 2008. http://dx.doi.org/10.1080/07900620701723570

FERREYRA, C.; LOË, R. C.; KREUTZWISER, R. D. Imagined communities, contested watersheds: Challenges to integrated water resources management in agricultural areas. Journal of Rural Studies, v. 24, n. 3, p. 304-321, 2008. http://dx.doi.org/10.1016/j.jrurstud.2007.11.001 
GADDIS, E. J. B.; FALK, H. H.; GINGER, C.; VOINOV, A. Effectiveness of a participatory modeling effort to identify and advance community water resource goals in St. Albans, Vermont. Environmental Modelling \& Software, v. 25, n. 11, p. 1428-1438, 2010. http://dx.doi.org/10.1016/j.envsoft.2009.06.004

HAJKOWICZ, S. Allocating scarce financial resources across regions for environmental management in Queensland, Australia. Ecological Economics, v. 61, n. 2-3, p. 208-216, 2007. http://dx.doi.org/10.1016/j.ecolecon.2006.10.011

HOPE, R. A. Evaluating Social Impacts of Watershed Development in India. World $\begin{array}{lllllll}\text { Development, } & \text { v. } 35, \quad \text { n. } & 8, & \text { p. }\end{array}$ http://dx.doi.org/10.1016/j.worlddev.2007.04.006

INSTITUTO BRASILEIRO DE GEOGRAFIA E ESTATÍSTICA - IBGE. Website. 2013. Disponível em: http://www.ibge.gov.br. Acesso em: 15 set. 2014.

KAPLOWITZ, M. D.; WITTER, S. G. Agricultural and residential stakeholder input for watershed management in a mid-Michigan watershed. Landscape and Urban Planning, v. 84, n. 1, p. 20-27, 2008. http://dx.doi.org/10.1016/j.landurbplan.2007.06.004

KORFF, Y. V.; D'AQUINO, P.; DANIELL, K. A.; BIJLSMA, R. Designing participation processes for water management and beyond. Ecology and Society, v. 15, n. 3, p. 1-24, 2010. http://www.ecologyandsociety.org/vol15/iss3/art1/

LIU, B. M.; ABEBE, Y.; MCHUGH, O. V., COLLICK, A. S.; GEBREKIDAN, B.; STEENHUIS, T. S. Overcoming limited information through participatory watershed management: Case study in Amhara, Ethiopia. Physics and Chemistry of the Earth, Parts A/B/C, v. 33, n. 1-2, p. 13-21, 2008. http://dx.doi.org/10.1016/j.pce.2007.04.017

MAHMOUD, M. I.; GUPTA, H. V.; RAJAGOPAL, S. Scenario development for water resources planning and watershed management: Methodology and semi-arid region case study. Environmental Modelling \& Software, v. 26, n. 7, p. 873-885, 2011. http://dx.doi.org/10.1016/j.envsoft.2011.02.003

MALHEIROS, T. F.; PROTA, M. G.; RINCÓN, M. A. P. Participação comunitária e implementação dos instrumentos de gestão da água em bacias hidrográficas. Revista Ambiente \& Água, v. 8, n. 1, p. 98-118, 2013. http://dx.doi.org/10.4136/ambi-agua.970

PERKINS, P. E. Public participation in watershed management: International practices for inclusiveness. Physics and Chemistry of the Earth, Parts A/B/C, v. 36, n. 5-6, p. 204212, 2011. http://dx.doi.org/10.1016/j.pce.2010.02.004

PINTO, N. G. M.; CORONEL, D. A.; CONTE, B. P. Raqueamento da degradação ambiental nos estados e regiões brasileiras. Revista Reunir, v. 6, n. 2, p. 68-82, 2016. http://dx.doi.org/10.18696/reunir.v6i2.374.

PORTO, M.; LA LAINA PORTO, R.; AZEVEDO, L. G. T. A participatory approach to watershed management: The Brazilian system. JAWRA Journal of the American Water Resources Association, v. 35, n. 3, p. 675-683, 1999. http://dx.doi.org/10.1111/j.1752-1688.1999.tb03623.x

PORTO, M. F. A.; PORTO, R. La L. Gestão de bacias hidrográficas. Estudos Avançados, V. 22, n. 63, p. 43-60, 2008. https://dx.doi.org/10.1590/S0103-40142008000200004 
PROGRAMA DAS NAÇÕES UNIDAS PARA O DESENVOLVIMENTO - PNUD; INSTITUTO DE PESQUISA ECONÔMICA APLICADA - IPEA; FUNDAÇÃO JOÃO PINHEIRO. Atlas do desenvolvimento humano no Brasil: ranking - todo o

Brasil. 2010. Disponível em: http://atlasbrasil.org.br/2013/pt/ranking. Acesso em: 15 set. 2014.

SANCHEZ-ROMAN, R. M.; FOLEGATTI, M. V.; ORELLANA GONZALEZ, A. M. G.; SILVA, R. T. da. Dynamic systems approach assess and manage water resources in river

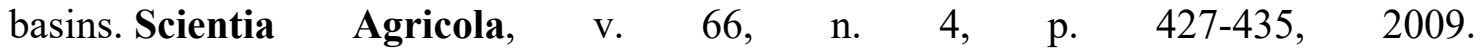
http://dx.doi.org/10.1590/S0103-90162009000400001

SAID, A.; SEHLKE, G.; STEVENS, D.K.; GLOVER, T.; SORENSEN, D.; WALKER, W.; HARDY, T. Exploring an innovative watershed management approach: From feasibility to sustainability. Energy, v. 31, n. 13, p. 2373-2386, 2006. http://dx.doi.org/10.1016/j.energy.2006.02.002

SISTEMA IBGE DE RECUPERAÇÃO AUTOMÁTICA - SIDRA. Website. 2014. Disponível em: http://www.sidra.ibge.gov.br/. Acesso em: 10 set. 2014.

SILVA, N. S.; RIBEIRO, C. A. A. S.; BARROSO, W. R.; RIBEIRO, P. E. Á.; SOARES, V. P.; SILVA, E. Sistema de Otto-codificação modificado para endereçamento de redes hidrográficas. Revista Árvore, v. 32, n. 5, p. 891-897, 2008. http://producao.usp.br/handle/BDPI/4386

TRATABRASIL. Saneamento no Brasil. 2014. Disponível em: http://www.tratabrasil.org.br/saneamento-no-brasil. Acesso em: 15 dez. 2014.

UNITED NATIONS DEVELOPMENT PROGRAMME - UNDP. Human Development Index (HDI). 2014. Disponível em: http://hdr.undp.org/en/content/human-developmentindex-hdi. Acesso em: 01 set. 2014.

WATANABE, M. M.; MADRUGA, L. R. G.; YAMAGUCHI, C. K.; VIEIRA, A. C. P.; JENOVEVA-NETO, R. Decision Making and Social Learning: the Case of Watershed Committee of the State of Rio Grande do Sul, Brazil. Water resources management, v. 28, n. 11, p. 3815-3828, 2014. http://dx.doi.org/10.1007/s11269-014-0711-2

WITTE, R. S.; WITTE, J. S. Estatística. 7. ed. Rio de Janeiro: LTC, 2005. 486 p. 